\title{
LitDB - Keeping Track of Research Papers From Your Institute Made Simple
}

\author{
Jörn Bethune ${ }^{1 \dagger}$, Lars Kraemer ${ }^{1 \dagger}$, Ingo Thomsen ${ }^{1}$, Andreas Keller ${ }^{2}$, David Ellinghaus ${ }^{1 \dagger}$ and Andre Franke ${ }^{1 * \dagger}$ (I)
}

\begin{abstract}
Background: In science peer-reviewed publications serve as an important indicator of scientific excellence and productivity. Therefore, every scientist and institution must carefully maintain and update records of their scientific publications. However, in most institutions and universities articles are often managed in a redundant file-based and non-central way. Whereas excellent reference management software packages such as Zotero, Endnote or Mendeley exist to manage bibliographies and references when writing scientific articles, we are not aware of any open source database solution keeping track of publication records from large scientific groups, entire institutions and/or universities.

Results: We here describe LitDB, a novel open source literature database solution for easy maintenance of publication lists assigned to various topics. In the last 2 years more than 50 users have been using LitDB at our research institute. The LitDB system is accessed via a web browser. Publications can be uploaded through direct exports from reference manager libraries or by entering PubMed IDs. Single users or user groups can track their citation counts, h-index and impact factor statistics and gain insights into the publication records of other users. It offers various visualization functions like coauthor networks and provides ways to organize publications from dedicated projects and user groups. The latter is in particular beneficial to manage publication lists of large research groups and research initiatives through a "crowd-sourcing" effort.

Conclusions: Keeping track of papers authored and published by a research group, institute or university is an important and non-trivial task. By using a centralized web-based platform for publication management such as LitDB the compilation of project- and group-related publication lists becomes easily manageable and it is less likely that papers are forgotten along the way.
\end{abstract}

Keywords: Management of project-related publication lists, Database, Web browser interface, Impact factor, Citations, Open source

\section{Background}

Scientific publications play an important role in research. The publication record is often used as an objective measure to benchmark productivity and excellence of a scientist within a research community. But modern scientists rarely work alone. They are part of research groups, academic departments or research initiatives. On each of these levels it is necessary to maintain current lists of publications to acquire third-party funding and to present a profile to the public.

\footnotetext{
* Correspondence: a.franke@mucosa.de

${ }^{\dagger}$ Equal contributors

${ }^{1}$ Institute of Clinical Molecular Biology, Christian-Albrechts-University of Kiel,

Kiel, Germany

Full list of author information is available at the end of the article
}

Funding applications are usually written by senior researchers who are managing several scientists with less experience. The senior researchers not only need to keep track of their own projects and publications, but also of all the work that is done by the junior researchers which can be a lot of projects running in parallel. Given the importance of publications in academia, it is undesirable to not know about the relevant publication of a paper from your own research institution.

Here we present a software-based solution to address this problem: We developed a literature management system called LitDB (Fig. 1). The LitDB software is a web-based application that allows researchers to store records of their own publications on a central server. This ensures that past publications are quickly 


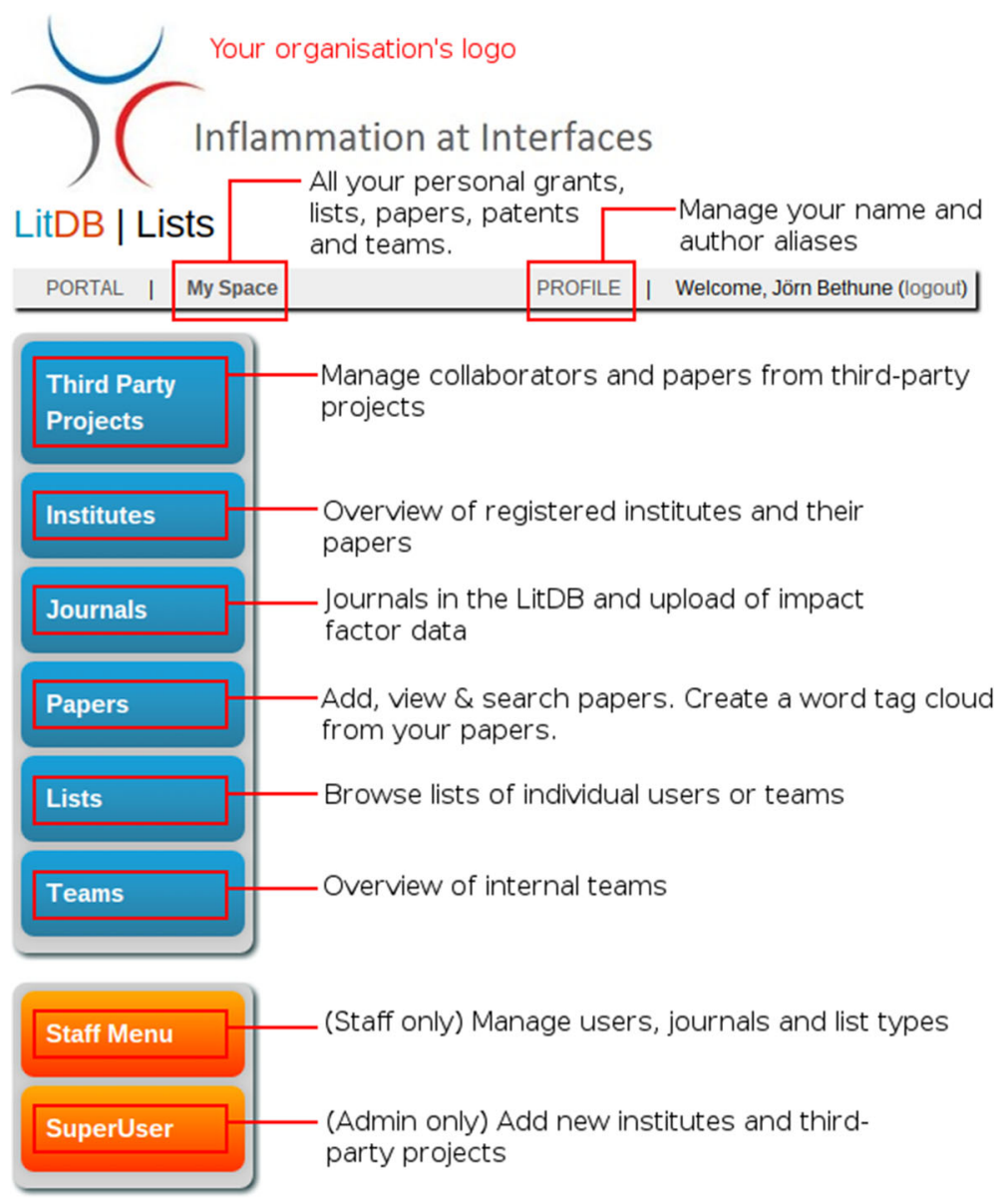

Fig. 1 LitDB functionality overview. The figure depicts all options that are available to a super user. Normal users only see the blue menu options and staff users only see the staff menu. All entries in the LitDB that are related to the current user can be accessed under My Space. It is possible to configure LitDB so that a different logo is shown

discoverable for everyone in a research organization and that publication lists for project-related grant applications can easily be constructed.

The crucial idea behind LitDB is that all members of a research institute use the same database to keep track of their own publications and their own projects, so that other members of a research institute are aware of all publications that can be used in research grant applications.

\section{Implementation}

LitDB is implemented in Python (version 2.7 [1]) with the Django web framework (version 1.8.7 [2]). It uses the PostgreSQL database [3] for data storage (via the python package psycopg2) and the D3.js [4] library for visualization of the co-author networks. LitDB has been tested on Microsoft Windows and Linux servers for the past 2 years. LitDB can make use of the open LDAP protocol (via the django-auth-ldap python package [5]) which is a standard for user management in most university's computer systems, but is also capable of managing users as a stand-alone tool.

We provide a Docker [6] image that facilitates the deployment of the LitDB so that it is not necessary to deal with the software's dependencies. For further technical information see the website of the LitDB: http://www.ikmb.uni-kiel.de/litdb.

\section{Results}

The central feature of LitDB is the management of project-related papers. Papers can be submitted manually, but LitDB assists the user in the process. For instance, it is possible to only provide the PubMed ID of a paper and LitDB will gather all the remaining metadata by itself. It is also possible to add several papers at once by providing a list of PubMed IDs. If no PubMed ID is available (e.g. for book chapters), the users can still enter their papers by providing the details themselves. Every user can list his or her own papers in LitDB (Fig. 2). Further papers that the user might have coauthored are also suggested. However, 


\begin{tabular}{|c|c|c|c|c|c|c|}
\hline \multicolumn{5}{|c|}{$\begin{array}{l}\text { Search: } \\
\text { You may enter multiple values separated by ', into one search field. The field will always use '', 'as search condition. } \\
\text { When using the 'LKE' or "LIKE" condition, use "' as wildcard for zero or more characters and '-' for a single character. } \\
\text { The "LIKE" condition adds wildcarts on both sides automatically. }\end{array}$} & \multirow{3}{*}{\multicolumn{2}{|c|}{$\square$ Extended }} \\
\hline WHERE & *LIKE* & & & & & \\
\hline \multicolumn{5}{|c|}{ parameters } & & \\
\hline \multicolumn{7}{|c|}{260 paper(s) } \\
\hline & Title & Journal & Year & MyPos & PMID & \\
\hline 20 & $\begin{array}{l}1000 \text { Genomes-based imputation identifies novel and refined associations for the Wellcome Trust } \\
\text { Case Control Consortium phase } 1 \text { Data. Jie Huang et al. }\end{array}$ & Eur. J. Hum. Genet. & 2012 & 3 & $\underline{22293688}$ & \\
\hline 20 & 15q13.3 microdeletions increase risk of idiopathic generalized epilepsy. Ingo Helbig et al. & Nat. Genet. & 2009 & 6 & 19136953 & \\
\hline 20 & $\begin{array}{l}\text { 16p11.2 } 600 \mathrm{~kb} \text { Duplications confer risk for typical and atypical Rolandic epilepsy. Eva M } \\
\text { Reinthaler et al. }\end{array}$ & Hum. Mol. Genet. & 2014 & 19 & $\underline{24939913}$ & \\
\hline 20 & $\begin{array}{l}\text { Abundant genetic overlap between blood lipids and immune-mediated diseases indicates shared } \\
\text { molecular genetic mechanisms. Ole A Andreassen et al. }\end{array}$ & PLOS ONE & 2015 & 11 & $\underline{25853426}$ & \\
\hline 20 & $\begin{array}{l}\text { A "candidate-interactome" aggregate analysis of genome-wide association data in multiple } \\
\text { sclerosis. Rosella Mechelli et al. }\end{array}$ & PLOS ONE & 2013 & banner & $\underline{23696811}$ & \\
\hline 20 & $\begin{array}{l}\text { A case-only study of gene-environment interaction between genetic susceptibility variants in NOD2 } \\
\text { and cigarette smoking in Crohn's disease aetiology. Katherine L Helbig et al. }\end{array}$ & BMC MED GENET & 2012 & 7 & $\underline{22416979}$ & \\
\hline 20 & $\begin{array}{l}\text { Accurate variant detection across non-amplified and whole genome amplified DNA using targeted } \\
\text { next generation sequencing. Abdou ElSharawy et al. }\end{array}$ & BMC GENOMICS & 2012 & last author & $\underline{22994565}$ & \\
\hline 20 & $\begin{array}{l}\text { A characterization in childhood inflammatory bowel disease, a new population-based inception } \\
\text { cohort trom South-Eastern Norway, 2005-07, showing increased incidence in Crohn's disease. } \\
\text { Gori Perminow et al. }\end{array}$ & Scand. J. Gastroenterol. & 2009 & 4 & 19117240 & \\
\hline 20 & $\begin{array}{l}\text { A common atopy-associated variant in the Th2 cytokine locus control region impacts transcriptional } \\
\text { regulation and alters SMAD3 and SP1 binding. A Kretschmer et al. }\end{array}$ & Allergy & 2014 & 11 & $\underline{24661001}$ & \\
\hline 20 & A common biological basis of obesity and nicotine addiction. TE Thorgeirsson et al. & Transl Psychiatry & 2013 & banner & $\underline{24084939}$ & \\
\hline 20 & $\begin{array}{l}\text { A common variant on chromosome } 11 q 13 \text { is associated with atopic dermatitis. Jorge Esparza- } \\
\text { Gordillo et al. }\end{array}$ & Nat. Genet. & 2009 & 14 & 19349984 & \\
\hline
\end{tabular}

Fig. 2 Listing of all papers from an author in the My Space section. With the help of the search form it is possible to search for specific papers. The overview table features the publication year and the author's position for every publication. It is possible to edit entries to change incorrect information

these papers must have been previously submitted by another LitDB user. The user can then acknowledge or reject the suggested papers. In order for the LitDB to recognize the papers of the users, every user can provide aliases for his or her name. For instance, PubMed usually only saves the first letter of the first name of a person. But sometimes the complete first name is available for the search. Therefore, it makes sense to search for "John Doe" as well as for "J Doe". This makes it also possible to take into account that people change names due to marriage, or to account for middle names or other circumstances. The problem of uniquely identifying scientists by their name(s) is not new. One popular solution to this problem is the use of unique IDs like ORCIDs [7]. The LitDB software also supports searching for papers by ORCID. Another useful feature is the possibility to categorize papers in the form of lists. When writing a grant application, it might be helpful to have a list that focuses on a specific scientific topic that is most relevant to the application that can be shared by several users. But other types of lists can also be created:
A list of the top 5 publications of a user is a popular choice and shown on our institute's webpage (with the help of an external script which queries the database). Lists can be created for single users but it is also possible to define projects in the LitDB and then all members of a project can create shared lists.

LitDB makes it possible to define working groups (teams), institutes and third-party projects so that all publications from a group of people can easily be obtained and employed for benchmarking, for example.

As it was demanded by the users, we implemented in LitDB the additional feature to record research prizes, third-party grants and granted patents (Fig. 3), all of which are important benchmarking features as well and therefore often needed in grant applications and CVs.

LitDB also provides a table with statistics on the number of published papers and the impact factors for each year. This table can be used for writing annual reports and to compare the paper output of different years with each other. 


\begin{tabular}{|c|c|c|c|c|c|c|c|c|}
\hline \multicolumn{2}{|c|}{ Grants (0) } & Lists (1) & Papers (260) & Patents (6) & Teams (6) & & & \\
\hline \multicolumn{9}{|c|}{ edit alias get PubMed Cltations } \\
\hline \multicolumn{9}{|c|}{ Impact Factor overview: } \\
\hline Year & Papers & & First author & Last author & IFAVG & IF MIN & IF $\operatorname{MAX}$ & IF SUM \\
\hline 2005 & 1 & & 0 & 0 & 29.648 & 29.648 & 29.648 & 29.648 \\
\hline 2006 & 4 & & 2 & 0 & 8.299 & 3.637 & 13.319 & 33.196 \\
\hline 2007 & 10 & & 3 & 0 & 12.276 & 3.534 & 29.648 & 122.759 \\
\hline 2008 & 5 & & 3 & 0 & 27.197 & 13.926 & 33.116 & 135.986 \\
\hline 2009 & 20 & & 0 & 2 & 8.336 & 2.113 & 29.648 & 158.380 \\
\hline 2010 & 32 & & 3 & 7 & 12.794 & 2.190 & 42.351 & 409.402 \\
\hline 2011 & 31 & & 2 & 2 & 16.803 & 2.450 & 54.420 & 520.882 \\
\hline 2012 & 38 & & 0 & 8 & 12.108 & 1.983 & 42.351 & 447.988 \\
\hline 2013 & 40 & & 0 & 8 & 10.469 & 2.190 & 29.648 & 418.741 \\
\hline 2014 & 31 & & 0 & 6 & 8.775 & 0.271 & 42.351 & 272.022 \\
\hline 2015 & 44 & & 0 & 8 & 13.945 & 2.316 & 42.351 & 599.626 \\
\hline 2016 & 1 & & 0 & 0 & 10.742 & 10.742 & 10.742 & 10.742 \\
\hline Total: & 257 & & 13 & 41 & 14.283 & 0.271 & 54.42 & 3159.372 \\
\hline \multicolumn{9}{|c|}{ download paper list } \\
\hline \multicolumn{9}{|c|}{ h-Index: 43 (PubMed Citations, last update: Jan. 10, 2016, 4:15 p.m.) } \\
\hline \multicolumn{9}{|c|}{$\begin{array}{l}\text { Fig. } 3 \text { My Space section and paper overview. The user can create and manage grants, lists of papers, all of his papers, patents and teams in } \\
\text { this section. In the current view, there is a table with yearly reports on the published papers. The number of papers, the number of first- and } \\
\text { last-authorships as well as the average, minimum, maximum and total impact factors for every year are listed }\end{array}$} \\
\hline
\end{tabular}

\section{Alternatives}

We have created LitDB because it provides a solution to a problem that we could not solve with other methods and non-commercial products. Most importantly, it provides a central hub for all authored papers for a group of researchers.

Often people use Microsoft Excel sheets or reference managers for keeping track of their publications. However, these tools are not well-integrated into larger context of a scientific institution. By using a central system, the scientists directly share their knowledge about their publications with their principal investigators, which leads to more consistent and sustainable archival of such information and makes it less likely that a publication is missed when writing a research grant. To our knowledge there are no other systems available to the general public that specialize on this task like LitDB does. It should be noted that LitDB does not try to replace reference managers. Its main purpose is to create central registry of all papers from a limited group of authors. Unlike popular reference managers like Endnote, Mendeley and Zotero, LitDB has been designed from the ground up for collaborative publication management.

LitDB also supports the calculation of impact factors for the papers in the system which is also helpful for raising funding for further research.

As the LitDB is a web-based application the researchers do not need to install any software beyond a web browser and everyone is able to work together with the same database, because there are no interoperability conflicts.

\section{Conclusions}

Keeping track of the scientific publications of a larger group of researchers requires a lot of effort and diligence in order to not forget any publications that might be relevant for receiving further funding. The LitDB assists a research group with managing publication records based on the principle of "crowd-sourcing" to keep publication records up-to-date and complete.

These publication records are also very valuable for performing benchmarking calculations in combination with impact factors to measure and report scientific success over the years.

\section{Availability and requirements \\ Project name: LitDB \\ Project home page: http://www.ikmb.uni-kiel.de/litdb \\ Operating system: GNU/Linux \\ Programming language: Python (version 2)}

Other requirements: A mod-wsgi compatible web server like Apache. Development libraries for libldap2, libsasl2 and libpq. The python packages django-auth-ldap and psycopg2. The Django web framework version 1.8.7

Licence: BSD License 2.0 


\section{Acknowledgements}

The authors are supported by the German Ministry of Education and Research (BMBF), the Deutsche Forschungsgemeinschaft (DFG) the Cluster of Excellence "Inflammation at Interfaces" and the DFG PhD Research Training Group"Genes, Environment and Inflammation" 1743/1. Andre Franke receives an endowment professorship (Peter Hans Hofschneider Professorship) of the "Stiftung Experimentelle Biomedizin" located in Zuerich, Switzerland.

\section{Funding}

Not applicable.

\section{Availability and requirements}

The source code and the most recent version of the LitDB tool can be obtained on the website http://www.ikmb.uni-kiel.de/litdb. The web server for the installation has to meet the following minimum requirements: 300 MB RAM, 600 MB disk space plus further space for the PostgreSQL database. The web interface has been tested with the following browsers: Safari 8.0.7 (Mac OS 10.10.4), Firefox 42.0 (Ubuntu Linux 14.04 LTS, Microsoft Windows 7)

\section{Author's contributions}

LK designed and developed the tool with support of IT. JB helped with the implementation and the release of the software package. AF initiated the project and supervised the development. JB and AF wrote the first draft of the manuscript. All authors read, edited and approved the final manuscript.

\section{Competing interests}

The authors declare that they have no competing interests. The authors alone are responsible for the content and writing of the paper.

\section{Consent for publication}

All personal data is from the authors and they give their consent to publish it.

\section{Ethics approval and consent to participate}

Not applicable.

\section{Publisher's Note}

Springer Nature remains neutral with regard to jurisdictional claims in published maps and institutional affiliations.

\section{Author details}

${ }^{1}$ Institute of Clinical Molecular Biology, Christian-Albrechts-University of Kiel, Kiel, Germany. ${ }^{2}$ Chair for Clinical Bioinformatics, Saarland University,

Saarbrücken, Germany.

Received: 25 April 2016 Accepted: 14 March 2017

Published online: 21 March 2017

\section{References}

1. Welcome to Python.org http://www.python.org. Accessed 21 Jan 2016

2. The Web framework for perfectionists with deadlines https://www. djangoproject.com/. Accessed 21 Jan 2016

3. PostgreSQL: The world's most advanced open source database http://www. postgresql.org/. Accessed 21 Jan 2016

4. D3.js - Data-Driven Documents http://d3js.org/ Accessed 21 Jan 2016

5. django-auth-ldap 1.2.7 : Python Package Index https://pypi.python.org/pypi/ django-auth-Idap/1.2.7. Accessed 21 Jan 2016

6. Docker - Build, Ship, and Run Any App, Anywhere https://www.docker com/. Accessed 21 Jan 2016

7. ORCID | Connecting Research and Researchers https://orcid.org/. Accessed 12 Feb 2017 